\title{
Odd-Even Layer-Number Effect and Layer-Dependent Magnetic Phase Diagrams in $\mathrm{MnBi}_{2} \mathbf{T e}_{4}$
}

\author{
Shiqi Yang, ${ }^{1,2,3, *}$ Xiaolong Xu ${ }^{1, *}$ Yaozheng Zhu, ${ }^{1, *}$ Ruirui Niu, ${ }^{1}$ Chunqiang $\mathrm{Xu},{ }^{4,5}$ Yuxuan Peng, ${ }^{1}$ Xing Cheng, \\ Xionghui Jia, ${ }^{1}$ Yuan Huang $\odot,{ }^{6, \dagger}$ Xiaofeng Xu, ${ }^{4}$ Jianming Lu, ${ }^{1, \$}$ and Yu Ye® ${ }^{1,2,7, \S}$ \\ ${ }^{1}$ State Key Laboratory for Mesoscopic Physics and Frontiers Science Center for Nano-optoelectronics, \\ School of Physics, Peking University, Beijing 100871, China \\ ${ }^{2}$ Collaborative Innovation Center of Quantum Matter, Beijing 100871, China \\ ${ }^{3}$ Academy for Advanced Interdisciplinary Studies, Peking University, Beijing 100871, China \\ ${ }^{4}$ Department of Applied Physics, Zhejiang University of Technology, Hangzhou 310023, China \\ ${ }^{5}$ School of Physics and Key Laboratory of MEMS of the Ministry of Education, \\ Southeast University, Nanjing 211189, China \\ ${ }^{6}$ Institute of Physics, Chinese Academy of Sciences, Beijing 100190, China \\ ${ }^{7}$ Beijing Key Laboratory for Magnetoelectric Materials and Devices, Beijing 100871, China
}

(Received 28 June 2020; revised 5 November 2020; accepted 23 November 2020; published 6 January 2021)

\begin{abstract}
Recently reported with nontrivial topological properties and magnetic orders, $\mathrm{MnBi}_{2} \mathrm{Te}_{4}$ is an intrinsic, magnetic topological insulator which holds promise for exploring exotic quantum phenomena such as the quantum anomalous Hall effect. However, the layer-dependent magnetism of $\mathrm{MnBi}_{2} \mathrm{Te}_{4}$, which is fundamental and crucial for further exploration of related quantum phenomena in this system, remains elusive. Here, by using polar reflective magnetic circular dichroism spectroscopy, we show that few-layered $\mathrm{MnBi}_{2} \mathrm{Te}_{4}$ exhibits an evident odd-even layer-number effect, i.e., the oscillations of the coercivity of the hysteresis loop (at $\mu_{0} H_{c}$ ) and the spin-flop transition (at $\mu_{0} H_{1}$ ), concerning the Zeeman energy and magnetic anisotropy energy. Noticeably, an anomalous magnetic hysteresis loop is observed in the evennumber septuple-layered $\mathrm{MnBi}_{2} \mathrm{Te}_{4}$, which might be attributed to the thickness-independent surfacerelated magnetization. A linear-chain model is applied to elucidate this odd-even layer-number effect of the spin-flop field and to determine the evolution of the magnetic states when subjected to an external magnetic field. A mean-field method further allows us to fully map the $\mathrm{MnBi}_{2} \mathrm{Te}_{4}$ flake's magnetic phase diagrams in the parameter space of the magnetic field, layer number, and, especially, temperature. By harnessing the unusual layer-dependent magnetic properties, our work paves the way for further study of quantum phenomena of $\mathrm{MnBi}_{2} \mathrm{Te}_{4}$.
\end{abstract}

DOI: $10.1103 /$ PhysRevX.11.011003

Subject Areas: Condensed Matter Physics, Magnetism

\section{INTRODUCTION}

Recently, the research on topological quantum materials has aroused tremendous interest and gained more and more attention in condensed matter physics [1-5]. Materials that combine magnetic and topological properties will reveal more exotic states, such as quantum anomalous Hall (QAH) insulators and axion insulators [6-9]. So far, such magnetic topological insulators (TIs) have been obtained by introducing magnetic atoms into TIs or using proximity

\footnotetext{
*These authors contributed equally to this work.

†yhuang01@iphy.ac.cn

\#jmlu@pku.edu.cn

${ }^{\S}$ ye_yu@pku.edu.cn
}

Published by the American Physical Society under the terms of the Creative Commons Attribution 4.0 International license. Further distribution of this work must maintain attribution to the author(s) and the published article's title, journal citation, and DOI. effects in magnetic and topological materials heterostructures, although the related exotic effects can only be observed at extremely low temperatures [6,10-12]. The recently discovered, layered $\mathrm{MnBi}_{2} \mathrm{Te}_{4}$, showing an out-ofplane easy axis with a ferromagnetic (FM) coupling within the layer and an antiferromagnetic (AFM) coupling between the adjacent layers (A type), is found to be an intrinsic magnetic TI with antiferromagnetism [13-18]. The effective combination of antiferromagnetic order and a nontrivial topological energy band makes $\mathrm{MnBi}_{2} \mathrm{Te}_{4}$ a promising material to discover novel topological phases and magnetic phase transitions by either controlling its crystal structure or applying magnetic fields [19-22]. Through complicated sample preparation processes, QAH and topological axion states are probed by lowtemperature electrical transport measurements in atomically thin flakes of $\mathrm{MnBi}_{2} \mathrm{Te}_{4}$ [23-25]. However, comprehensively revealing the magnetic phase transitions of $\mathrm{MnBi}_{2} \mathrm{Te}_{4}$ under varying external magnetic field, temperature, and number of layers has not been studied yet, which 
is of great significance for further exploration of the rich topological phenomena under different magnetic phases.

Polar reflective magnetic circular dichroism (RMCD) spectroscopy, which measures the differential absorption of left and right circularly polarized light induced by the out-of-plane magnetization of the sample (parallel to the light propagation), is a nondestructive optical method for measuring and imaging the magnetism of microsized flakes [26-28]. Owing to the small size of the laser spot (about $2 \mu \mathrm{m}$ in diameter), the RMCD spectroscopy measurement is less influenced by the inhomogeneity of the structure (the measured domain size of the $\mathrm{MnBi}_{2} \mathrm{Te}_{4}$ bulk is on the order of $10 \mu \mathrm{m}$ ) [29], enabling subtle magnetic phases originating from finite-size effects in few-number (few- $N$ ) septuple layer (SL) $\mathrm{MnBi}_{2} \mathrm{Te}_{4}$ flakes to be detected. Besides, the RMCD measurement does not require a complicated sample preparation process, which reduces fabrication-induced surface damage or doping effects, and is very suitable for layer-dependent magnetic studies.

In this work, we utilize the RMCD measurement (see the setup in Fig. S1 of the Supplemental Material, Sec. I [30]) to systematically study the magnetic properties of thin flakes, from single-SL to 9-SL, and 25-SL $\mathrm{MnBi}_{2} \mathrm{Te}_{4}$ under different applied magnetic fields and temperatures. The results show that for a single-SL sample, the ferromagnetism is retained, and as the number of layers increases, the Néel temperature $T_{N}$ of the antiferromagnetic arrangement in adjacent layers increases simultaneously (from $15.2 \mathrm{~K}$ of 1 -SL to $24.5 \mathrm{~K}$ of 25 -SL samples). The magnetic behavior of $\mathrm{MnBi}_{2} \mathrm{Te}_{4}$ exhibits an evident odd-even layer-number

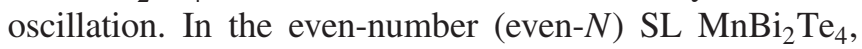
an anomalous magnetic hysteresis loop is observed, which is possibly attributed to the thickness-independent surface-related magnetization. Combining the experimentally observed spin-flop transitions with the odd-even effect and theoretical calculations of the linear-chain model, the dependence of the spin-flop field on the number of SLs also allows us to accurately determine the interlayer exchange coupling strength $(0.68 \mathrm{meV})$ and the magnetic anisotropy energy $(0.21 \mathrm{meV})$ for the layered $\mathrm{MnBi}_{2} \mathrm{Te}_{4}$, thus capturing the magnetic phase evolution under the external magnetic field. The mean-field (MF) method allows us to trace $\mathrm{MnBi}_{2} \mathrm{Te}_{4}$ flakes' magnetic phase diagrams, depending on the applied magnetic field, the number of layers, and the temperature. The phase boundaries obtained experimentally agree well with the theoretical calculations, revealing the capability of the MF model to trace the phase transitions in such two-dimensional (2D) antiferromagnetic materials. Our experimental and theoretical findings have determined the magnetic phase diagrams of $\mathrm{MnBi}_{2} \mathrm{Te}_{4}$ with the number of layers, the temperature, and the external magnetic field, keeping the promise for future exploration of quantum phenomena in this intrinsic magnetic TI by controlling its magnetic phases.

\section{EXPERIMENT AND ANALYSIS}

\section{A. Layer-dependent ferromagnetism}

$\mathrm{MnBi}_{2} \mathrm{Te}_{4}$ is a layered ternary tetradymite compound with space group $R \overline{3} m$ [31], which consists of Te-Bi-Te-Mn-TeBi-Te SL stacking through van der Waals (vdW) force. Below the $T_{N}$, the spins of $\mathrm{Mn}^{2+}$ ions couple ferromagnetically within the SL with an out-of-plane easy axis, but they have an antiferromagnetic exchange coupling with the adjacent SL [Fig. 1(a)], showing an A-type AFM order. The room-temperature Raman spectrum of the $\mathrm{MnBi}_{2} \mathrm{Te}_{4}$ crystal shows well-resolved $E_{g}\left(47 \mathrm{~cm}^{-1}\right), A_{1 g}\left(66 \mathrm{~cm}^{-1}\right)$, $E_{g}^{2}\left(104 \mathrm{~cm}^{-1}\right)$, and $A_{1 g}^{2}\left(139 \mathrm{~cm}^{-1}\right)$ Raman modes [see Fig. S3(a) in the Supplemental Material [30] ], consistent with previous reports $[17,32]$. The temperature-independent Raman spectra imply that there is no structure transition in the measured temperature range down to $2 \mathrm{~K}$ [see Fig. S2 and Fig. S3(b) in the Supplemental Material [30] ]. Atomically thin flakes down to $1 \mathrm{SL}$ are mechanically exfoliated from bulk crystals onto the gold substrates using the standard Scotch tape method and usually protected by a layer of polymethyl methacrylate (PMMA). Figures 1(b) and 3(a) display typical optical images of 1-SL and few-SL $\mathrm{MnBi}_{2} \mathrm{Te}_{4}$ samples, showing obvious optical contrasts in different thicknesses, which are confirmed by atomic force microscopy characterizations (see Fig. S4 in the Supplemental Material, Sec. III [30] for details). The height line profiles of the 1-SL [Fig. 1(c)] and the stepped $\mathrm{MnBi}_{2} \mathrm{Te}_{4}$ flakes [Fig. 3(b)] indicate a SL thickness of about $1.4 \pm 0.1 \mathrm{~nm}$, consistent with previous reports [17,22].

The magnetic order of few- $N$ SL $\mathrm{MnBi}_{2} \mathrm{Te}_{4}$ was probed by RMCD microscopy as a function of the applied external magnetic field perpendicular to the sample plane. The RMCD signals were collected under a $0.25-\mu \mathrm{W}, 633-\mathrm{nm}$ $\mathrm{HeNe}$ laser excitation with a spot size of about $2 \mu \mathrm{m}$ (see results under a 532-nm continuous-wave laser excitation in Fig. S5 of the Supplemental Material, Sec. IV [30]). Figure 1(d) shows the magnetic field dependence of the RMCD signals of 1-SL $\mathrm{MnBi}_{2} \mathrm{Te}_{4}$ in a temperature range from $1.6 \mathrm{~K}$ to $18 \mathrm{~K}$. The nonzero RMCD signal at zero field and a clear hysteresis loop confirms the ferromagnetism of 1-SL $\mathrm{MnBi}_{2} \mathrm{Te}_{4}$. As the temperature increases, the hysteresis loop shrinks and disappears at $18 \mathrm{~K}$, indicating a FM to paramagnetic $(\mathrm{PM})$ phase transition.

To study the layer-dependent magnetism, we investigate the behavior of thin flakes from 1 SL to 9 SLs under a magnetic field sweeping back and forth from $+7 \mathrm{~T}$ to $-7 \mathrm{~T}$ at $1.6 \mathrm{~K}$. RMCD signals versus $\mu_{0} H$ are shown in Fig. 1(e). All measured odd-number (odd- $N$ ) SLs consistently show a FM behavior with a single hysteresis loop centered at $\mu_{0} H=0 \mathrm{~T}$, highlighted by the grey shaded area in Fig. 1(e), indicating its ferromagnetic feature due to an uncompensated layer. The coercive field $\mu_{0} H_{c}^{\text {odd }}$ increases monotonously with the thickness. In an odd- $N$ SL A-type AFM material, the Zeeman energy at a fixed magnetic field 

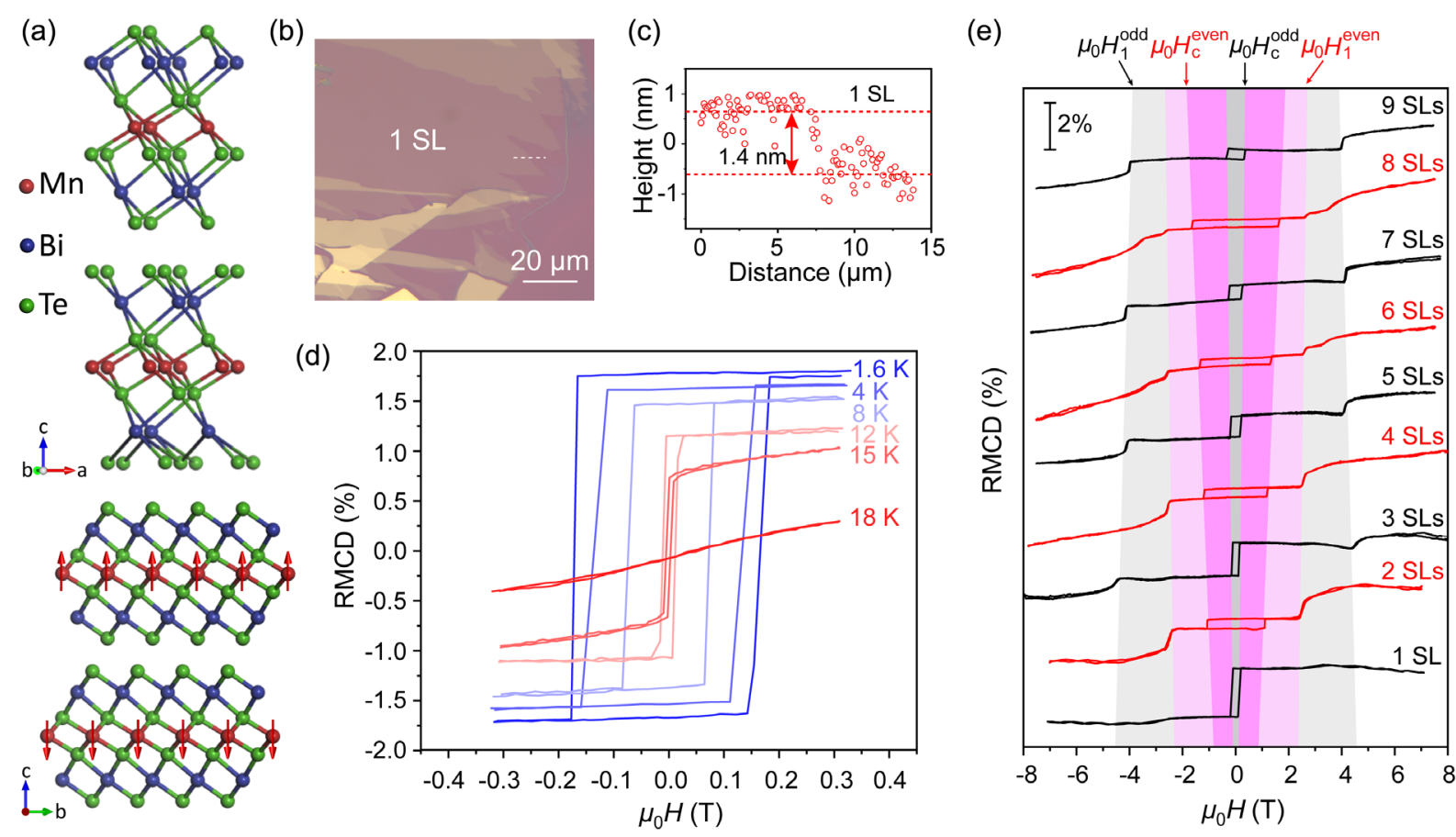

FIG. 1. Crystal structure and RMCD measurements of 1-SL to 9-SL $\mathrm{MnBi}_{2} \mathrm{Te}_{4}$ flakes. (a) Crystal structure of $\mathrm{MnBi}_{2} \mathrm{Te}_{4}$. The septuple atomic layers are stacked through vdW force. The arrows on the Mn atoms denote the magnetic moment of each Mn ion. Without an external magnetic field, the neighboring ferromagnetic SLs couple antiferromagnetically, with an out-of-plane orientation. (b) Typical optical image of a 1-SL $\mathrm{MnBi}_{2} \mathrm{Te}_{4}$ on the gold substrate. (c) Height line profile of the single-SL MnBi $\mathrm{Te}_{4}$, along with the white dashed line in panel (b). The step height is $1.4 \mathrm{~nm}$, consistent with the thickness of 1-SL $\mathrm{MnBi}_{2} \mathrm{Te}_{4}$. (d) Temperature-dependent RMCD measurements of the 1-SL $\mathrm{MnBi}_{2} \mathrm{Te}_{4}$. (e) $\mathrm{RMCD}$ measurements of $\mathrm{MnBi}_{2} \mathrm{Te}_{4}$ flakes (from $1 \mathrm{SL}$ to 9 SLs) at $1.6 \mathrm{~K}$. The shaded areas highlight the thickness dependence of the low-field spin-flip and spin-flop phase transitions in odd- $N$ and even- $N$ SL samples. Note that the layer-dependent measurements are performed on the freshly exfoliated flake without PMMA coating.

is proportional to the single uncompensated SL magnetization (invariant with the film thickness), while the anisotropy energy is the sum of that of all SLs (adds up the film thickness). Thus, a higher magnetic field is required for the Zeeman energy to overcome the anisotropy energy in the thicker odd- $N$ SL materials, resulting in a larger coercive field $\mu_{0} H_{c}^{\text {odd }}$. Surprisingly, we also observe an anomalous magnetic hysteresis loop centered at $\mu_{0} H=0 \mathrm{~T}$ in even- $N$ $\mathrm{SL} \mathrm{MnBi}_{2} \mathrm{Te}_{4}$, indicating a net magnetization, which is unexpected for an A-type AFM material. We note that this anomalous FM response was also observed in Hall resistance measurements of 4-SL $\mathrm{MnBi}_{2} \mathrm{Te}_{4}$, and it was attributed to the possible substrate-induced top-bottom surface asymmetry or disorders in the sample [23]. The observed magnetic hysteresis loop is persistent in all the measured even- $N$ SL $\mathrm{MnBi}_{2} \mathrm{Te}_{4}$, and its coercive field, $\mu_{0} H_{c}^{\text {even }}$ [highlighted by the pink shaded area in Fig. 1(e)], also increases with the film thickness like in odd- $N$ SL samples. Thus, we speculate that the net magnetization observed in the even- $N$ SL samples is a thickness-independent surfacerelated magnetization, while the thickness-dependent (such as impurities, defects, or disorders) magnetization will increase with the layer number, resulting in the insensitivity of $\mu_{0} H_{c}^{\text {even }}$ to the layer number, which contradicts our observation. Based on the fact that $\mu_{0} H_{c}^{\text {even }}$ is much larger than $\mu_{0} H_{c}^{\text {odd }}$ regardless of the film thickness, we can also conclude that the net magnetization in the even- $N$ SL samples is much smaller than that in the odd- $N$ SL samples.

Under larger magnetic fields, both the odd- $N$ (except for the $1 \mathrm{SL}$ ) and even- $N$ SL flakes undergo spin-flop transitions and evolve into complete out-of-plane magnetization above the spin-flip transition fields $\left(\mu_{0} H_{2}\right)$ [14,17,33]. However, for the flakes with $N \geq 4$, the spin-flip fields are too large that exceed the magnitude of the magnetic field we apply [23-25]. The spin-flop transitions exhibit a strong odd-even layer-number effect. The spin-flop fields $\left(\mu_{0} H_{1}\right)$ in the odd- $N$ SL flakes are much larger than those in the even- $N$ SL flakes, and they decrease (slightly increase) with the film thickness in the odd- $N$ (even- $N$ ) SL samples.

\section{B. Spin-flop transitions with odd-even effects and linear-chain model}

For all measured odd- $N$ SL (3-SL, 5-SL, 7-SL, and 9-SL) $\mathrm{MnBi}_{2} \mathrm{Te}_{4}$ samples, a spin-flop transition at about $4.5 \mathrm{~T}$ is observed [Fig. 2(a)]. The spin-flop field of the measured odd$N$ flakes $\left(\mu_{0} H_{1}^{\text {odd }}\right)$ decreases as the number of layers increases [green circles and corresponding error bars in Fig. 2(b)]. In contrast, the spin-flop transition in the measured even- $N$ SL (2-SL, 4-SL, 6-SL, and 8-SL) $\mathrm{MnBi}_{2} \mathrm{Te}_{4}$ samples occurs at a 

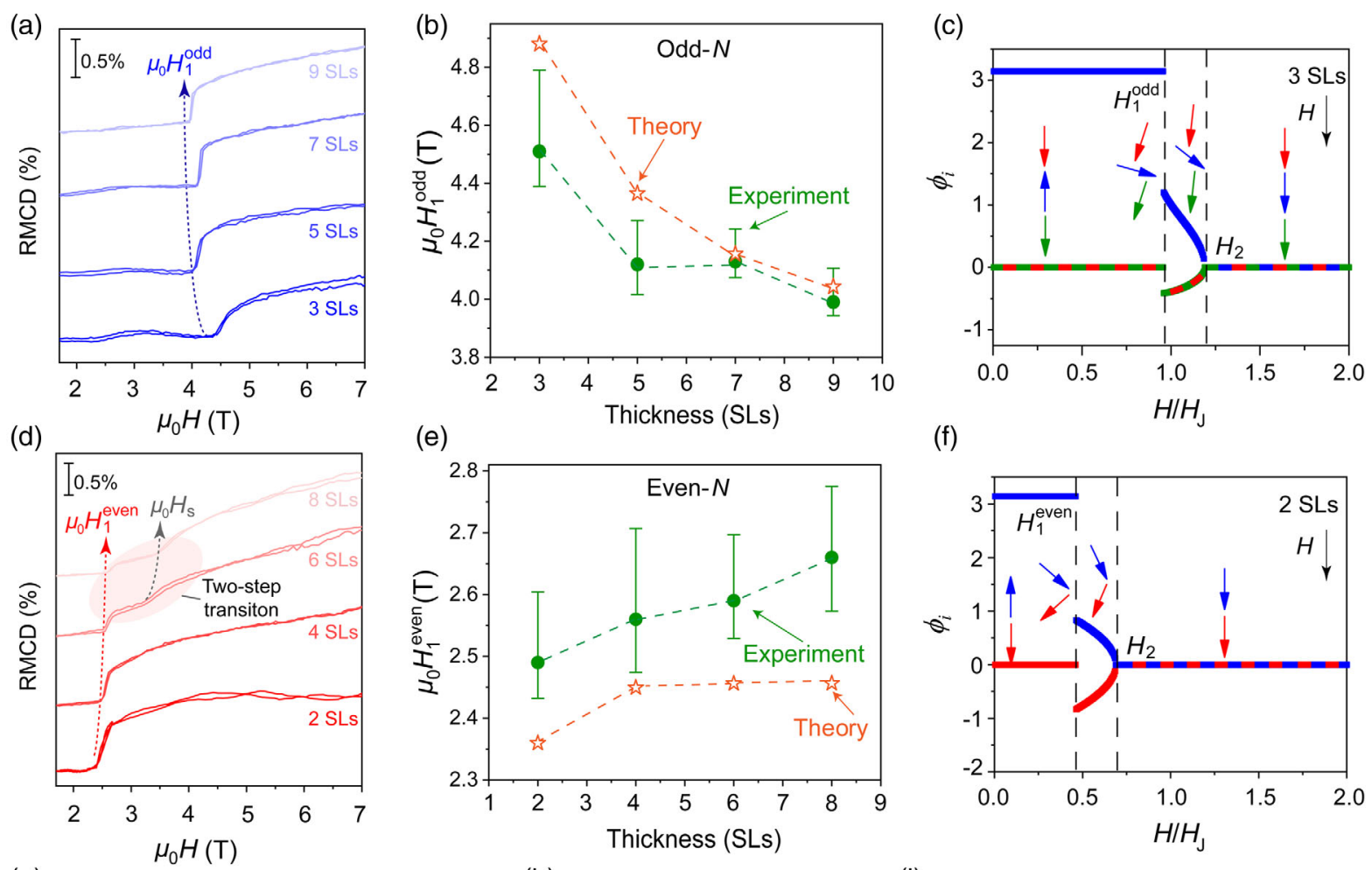

(f)
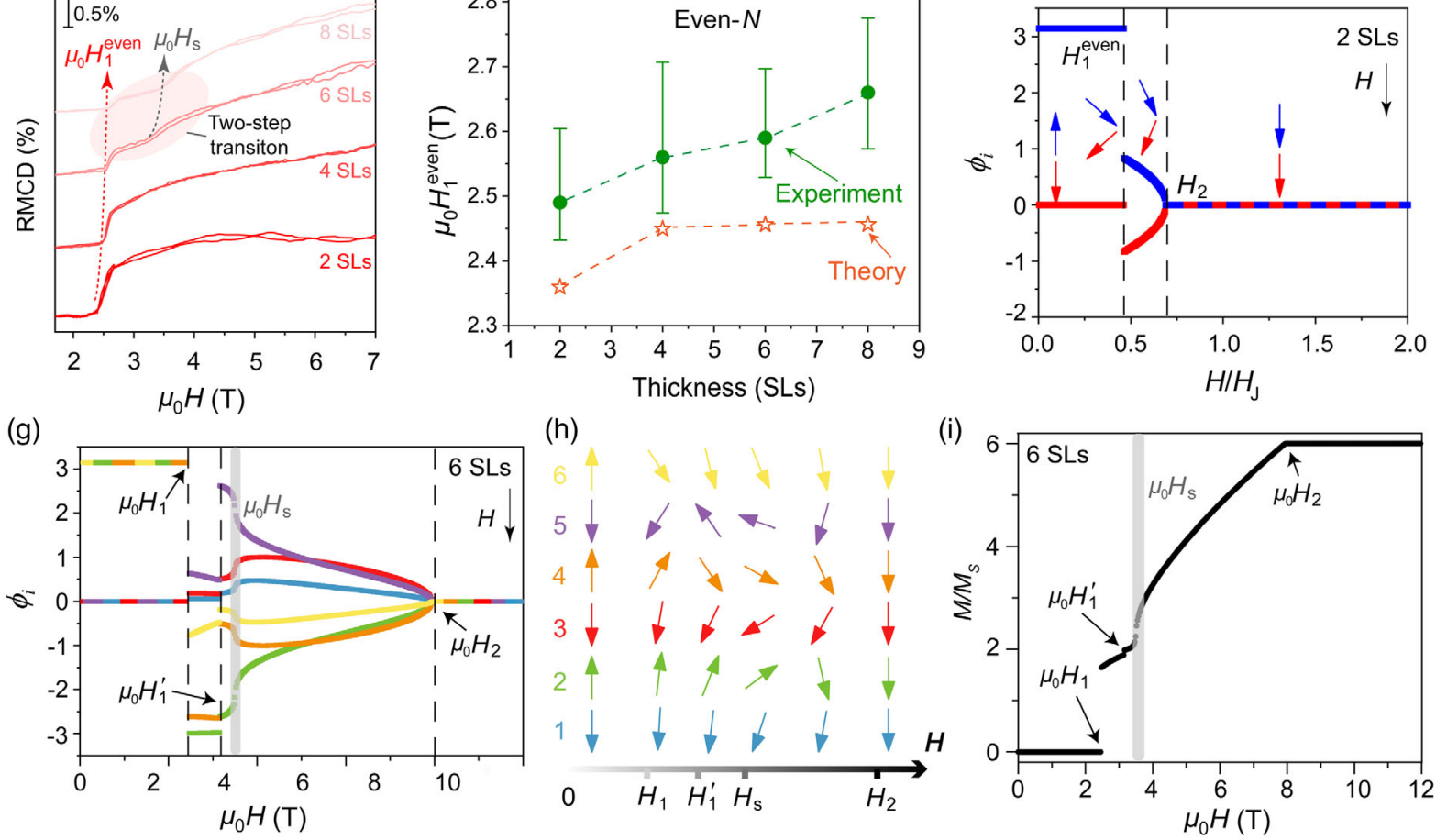

FIG. 2. Odd-even layer-number effect and magnetic-state evolution of thin $\mathrm{MnBi}_{2} \mathrm{Te}_{4}$ flakes. (a,d) Zoomed-in RMCD measurements of all investigated odd- $N$ samples (a) and even- $N$ samples (d), showing a prominent odd-even layer-number effect in $\mathrm{MnBi}_{2} \mathrm{Te}_{4}$. In odd- $N$ SLs, the spin-flop transition occurs at about $4.5 \mathrm{~T}$. The blue dashed arrow represents the spin-flop field in each thickness. In even- $N$ SLs, the spin-flop transition occurs at about $2.5 \mathrm{~T}$, which is marked by the red dashed arrow. For 6 SLs and 8 SLs, the RMCD signals exhibit a second transition marked by the grey dashed arrow. (b,e) Spin-flop field versus $N$ for odd- $N$ SL (b) and even- $N$ SL (e) samples. The green circles with error bars denote the experimental results extracted from the RMCD measurements, while orange pentagons denote the calculated theoretical values of spin-flop fields using the parameters of $\mu_{0} H_{J}=5.10 \mathrm{~T}$ and $\mu_{0} H_{K}=1.58 \mathrm{~T}$. The dashed lines indicate the evolution of the spin-flop field (green for experiment and orange for theory). For the even- $N$ samples, there is a small deviation of $\mu_{0} H_{1}^{\text {even }}$ between the theory and experiment, which may result from the nonzero magnetization in their ground AFM states at zero field. (c,f) Magnetic-state evolution under the applied magnetic field in 3-SL (c) and 2-SL (f) $\mathrm{MnBi}_{2} \mathrm{Te}_{4}$ obtained from the antiferromagnetic linear-chain model. Different colors denote the evolution of each layer in the 3-SL (c) and 2-SL (f) samples, and the arrow orientations indicate the detailed spin alignments. Dashed lines reveal the coincident evolution of different layers. (g) Magneticstate evolution of the 6-SL sample under external magnetic field. Different colors represent the spin arrangement of different layers. (h) Detailed spin alignments of layers 1 to 6, with the corresponding colors of ( $g$ ) at several representative external magnetic fields. (i) Calculated total magnetization along the $z$ axis (defined as $M=M_{s} \sum_{i=1}^{N} \cos \phi_{i}$ ) as a function of the external field for 6-SL $\mathrm{MnBi}_{2} \mathrm{Te}_{4}$. The magnetization change at $\mu_{0} H_{1}^{\prime}$ is almost invisible compared to that at $\mu_{0} H_{1}$ and the coherent increase at $\mu_{0} H_{s}$.

much smaller field of about $2.5 \mathrm{~T}$ [Fig. 2(d)], and the value of $\mu_{0} H_{1}^{\text {even }}$ increases slightly with the number of layers [Fig. 2(e)]. There is also a distinguishable second transition in RMCD measurements of 6-SL and 8-SL samples [highlighted by the grey dashed arrow in Fig. 2(d)], which stem from the sharp coherent spin rotations in a narrow magnetic field range (beginning at $\mu_{0} H_{s}$ ) after the multistep spin-flop transitions predicted in the theoretical model (see 
detailed description in the following). Compared with recently published transport works, the $\mu_{0} H_{1}$ obtained in our RMCD measurements does not coincide with the field at which the zero Hall plateau of $R_{x y}$ begins to deviate, but it is close to the beginning field of the magnetic-field-driven spin-flipping process observed in $R_{x x}$ [24,25]. Considering the topological nature, the relationship between the magnetic-state transition and transport behavior still needs more in-depth exploration. Besides, the fabrication-induced hole doping in the $\mathrm{MnBi}_{2} \mathrm{Te}_{4}$ flakes will shift the magnetic transition fields, which may also cause the difference between our measurements and the transport works. This prominent odd-even layer-number effect, with a magnetic phase transition occurring at respective finite fields for odd- $N$ and even$N$ SL flakes, is representative and useful to understand the origin in terms of the magnetic phases in layered antiferromagnets $\mathrm{MnBi}_{2} \mathrm{Te}_{4}$ under an applied magnetic field.

\section{Linear-chain model}

The magnetic phase transitions can be understood quantitatively using an antiferromagnetic linear-chain model, where the magnetization of each layer is represented by a "macro-spin" coupled to its nearest-neighbor layers through the interlayer exchange energy $J$. This simplification is effective when the intralayer ferromagnetic coupling is much stronger than the interlayer antiferromagnetic coupling [34], and it is reasonable to assume uniform magnetization within the single layer at zero temperature. Denoting the magnetization per unit cell in the $i$ th layer as $\vec{M}_{i}$, then the average energy per unit cell reads

$$
\begin{aligned}
U_{N}= & J \sum_{i=1}^{N-1} \frac{\vec{M}_{i} \cdot \vec{M}_{i+1}}{M_{s}^{2}}-\frac{K}{2} \sum_{i=1}^{N}\left(\frac{\vec{M}_{i} \cdot \hat{z}}{M_{s}}\right)^{2} \\
& -\mu_{0} \vec{H} \cdot \sum_{i=1}^{N} \vec{M}_{i},
\end{aligned}
$$

where $M_{s}$ is the saturation magnetization per unit cell of a single layer, $K>0$ is the easy-axis anisotropy energy, and $\vec{H}$ denotes the applied magnetic field. Here, the anisotropy includes both magnetocrystalline anisotropy $\left(K_{\mathrm{mc}}\right.$, from spin-orbit coupling in the material) and the shape anisotropy ( $K_{\mathrm{sh}}$, associated with magnetostatic interactions). Actually, $K_{\mathrm{sh}}=-\mu_{0} M_{s}^{2} / V$, where $V$ is the volume of the unit cell. However, $K_{\mathrm{mc}}$ is relatively large in $\mathrm{MnBi}_{2} \mathrm{Te}_{4}$, and $K=K_{\mathrm{mc}}+K_{\mathrm{sh}}$ is positive. At zero temperature, we can express that $\vec{M}_{i}=M_{s} \hat{e}_{i}$, where $\hat{e}_{i}$ is a unit vector. Then, the magnetic energy reads

$U_{N}=J \sum_{i=1}^{N-1} \hat{e}_{i} \cdot \hat{e}_{i+1}-\frac{K}{2} \sum_{i=1}^{N}\left(\hat{e}_{i} \cdot \hat{z}\right)^{2}-\mu_{0} M_{s} \vec{H} \cdot \sum_{i=1}^{N} \hat{e}_{i}$.
In our experiment, $\vec{H}$ is along the $z$ axis (out of plane). To minimize the energy $U_{N}$, all $\hat{e}_{i}$ must be on the same plane (perpendicular to the sample plane). Taking this plane as the $x z$ plane, the magnetization can be expressed as $\vec{M}_{i}=M_{s}\left(\sin \phi_{i}, 0, \cos \phi_{i}\right)$, where $\phi_{i}$ is the angle between the magnetization in the $i$ th layer and the $z$ axis. In terms of $\phi_{i}$, the magnetic energy reads

$$
\begin{aligned}
U_{N}= & J \sum_{i=1}^{N-1} \cos \left(\phi_{i}-\phi_{i+1}\right) \\
& -\frac{K}{2} \sum_{i=1}^{N}\left(\cos \phi_{i}\right)^{2}-\mu_{0} M_{s} H \sum_{i=1}^{N} \cos \phi_{i} .
\end{aligned}
$$

By defining the magnetic-field-scale parameters $H_{J}=$ $(2 J) /\left(\mu_{0} M_{s}\right)$ and $H_{K}=K /\left(\mu_{0} M_{s}\right)$, we obtain

$$
\begin{aligned}
U_{N}= & \mu_{0} M_{s}\left[\frac{H_{J}}{2} \sum_{i=1}^{N-1} \cos \left(\phi_{i}-\phi_{i+1}\right)\right. \\
& \left.-\frac{H_{K}}{2} \sum_{i=1}^{N}\left(\cos \phi_{i}\right)^{2}-H \sum_{i=1}^{N} \cos \phi_{i}\right] .
\end{aligned}
$$

See Supplemental Material, Sec. V, Parts I and III [30] for detailed information on the numerical solution of this model. This model works well for layered antiferromagnetic materials in three regions, namely, the low-anisotropy region $\left(H_{K} / H_{J}=0\right)$, the mid-anisotropy region $\left(H_{K} / H_{J}=0.3\right)$, and the high-anisotropy region $\left(H_{K} / H_{J}=0.6\right)$, as shown in Fig. S6 (see Supplemental Material, Sec. V, Part II [30] for details), which allows us to quantitatively analyze the $N$-dependent magnetic phase transitions observed in the $\mathrm{MnBi}_{2} \mathrm{Te}_{4}$ system.

\section{Spin-flop transition and magnetic-state evolution}

From this model, we first quantitatively explain the evolution of the spin-flop field with the thickness. According to the experimental values of the spin-flop field for each $N$ (from 2 to 9), we perform a standard $\chi^{2}$ fitting for $H_{J}$ and $H_{K}$, which indicates $\mu_{0} H_{J}$ to be $5.10 \mathrm{~T}$ and $\mu_{0} H_{K}$ to be $1.58 \mathrm{~T}$ (see Supplemental Material, Sec. V, Part III [30] for details). The theoretically predicted spin-flop fields agree well with the experimentally observed values in both odd- $N$ [Fig. 2(b)] and even- $N$ SL samples [Fig. 2(e)]. For the odd- $N$ SL samples, the magnetization of one uncompensated layer contributes a finite Zeeman energy to the total energy under the external magnetic field at the AFM state. Therefore, the spin-flop transition in odd- $N$ SL samples always occurs at a higher magnetic field than that in the even- $N$ SL samples, where no net magnetization at the AFM state is expected. It is worth noting that $\mu_{0} H_{1}^{\text {odd }}$ decreases with the thickness since this effect originates from the additional Zeeman contribution of one individual uncompensated layer competing against the energetic 
contribution of all layers [34]. For the even- $N$ SL samples, there is a small discrepancy of $\mu_{0} H_{1}^{\text {even }}$ between theory and experiment (the experimentally observed field values are, overall, slightly larger than those of the theoretical prediction), which may confirm the existence of the net magnetization in their ground AFM states at zero field, i.e., the possible surface-related magnetization discussed above. This nonzero magnetization could lead to an extra finite Zeeman term in the expression of AFM energy, which further reduces the energy of the AFM state and causes the increase in $\mu_{0} H_{1}^{\text {even }}$ (see Fig. S7 in the Supplemental Material, Sec. V, Part III [30] for the calculation considering a possible surface-related magnetization).

By utilizing the extracted values of $H_{J}$ and $H_{K}$, the evolution of the "macrospins" in each SL under an external magnetic field that unravels the nature of the magnetic phase transitions can be reproduced. For the 3-SL flake [Fig. 2(c)], the magnetizations in the adjacent layers remain antiparallel to each other along the $z$-axis direction until $\mu_{0} H$ reaches $\mu_{0} H_{1}^{\text {odd }}$ and undergoes a spin-flop transition to a canting AFM (CAFM) state. The magnetizations of the top and bottom layers experience the same spin-flop and canting processes under an external field. With a further increase of the magnetic field, the noncollinear magnetizations eventually reach a fully aligned FM state through coherent rotation at $\mu_{0} \mathrm{H}_{2}$. For the 2-SL flake [Fig. 2(f)], the magnetizations in the two layers remain antiparallel to each other until $\mu_{0} H$ reaches $\mu_{0} H_{1}^{\text {even }}$, and they experience a spin-flop transition to the CAFM state and eventually realize the FM state. The magnetic-state evolutions discussed for the 2-SL and 3-SL samples are characteristics for all even- $N$ and odd- $N$ SL samples.

Furthermore, this model also predicts a multistep spinflop transition in 4-SL, 6-SL, and 8-SL samples, where an asymmetric state is first formed at $\mu_{0} H_{1}$ [e.g., $\phi_{1} \neq-\phi_{6}$, $\phi_{2} \neq-\phi_{5}$, and $\phi_{3} \neq-\phi_{4}$ for $N=6$ in Figs. 2(g) and 2(h)]; then, the symmetric state is reached at $\mu_{0} H_{1}^{\prime}\left(\phi_{1}=-\phi_{6}\right.$, $\phi_{2}=-\phi_{5}$, and $\phi_{3}=-\phi_{4}$ for $N=6$ ), completing the spin-flop transition through multiple steps. In the asymmetric state $\left(H_{1}<H<H_{1}^{\prime}\right)$, the spins in layers 1 to 3 remain almost unchanged, while the spins in layers 4 to 6 flop to a prominent canting state. In the symmetric state $\left(H_{1}^{\prime}<H<H_{2}\right)$, the canting state is observed symmetrically in all layers. This multistep spin-flop process is probably coincident with the surface spin-flop (SSF) transition and bulk spin-flop transition discussed in a recently published work of thick $\mathrm{MnBi}_{2} \mathrm{Te}_{4}$ [35]. We note that a sharp coherent spin rotation that occurs within a narrow magnetic field range closely follows the final-step spin-flop transition in the 6-SL [indicated by the grey area in Fig. 2(g)] and 8-SL samples, but not in the 4-SL sample (see details in Fig. S8 and Fig. S9 in the Supplemental Material, Sec. V, Part IV [30]). Combined with the fact that the magnetization changes induced by the multistep spinflop transitions (apart from the change at $\mu_{0} H_{1}$ ) are remarkably small [Fig. 2(i)] in 6 and 8 SLs, we can conclude that the experimentally observed two-step transitions in 6-SL and 8-SL samples originate from the firststep spin-flop transition (at $\mu_{0} H_{1}$ ) and the subsequent sharp coherent spin rotation $\left(\right.$ at $\mu_{0} H_{s}$ ) that follows the multistep spin-flop transition process. Because of the good agreement between the theoretical and experimental results, we conclude that this model reproduces the layer number-dependent magnetic transitions using parameters of interlayer exchange energy $J=0.68 \mathrm{meV}$ and magnetic anisotropy energy $K / 2=0.21 \mathrm{meV}$. We also acquire the RMCD intensity maps including 3-SL, 4-SL, and 5-SL $\mathrm{MnBi}_{2} \mathrm{Te}_{4}$ flakes under specific external magnetic fields (see Fig. S10 in the Supplemental Material, Sec. VI [30] for details), revealing that the evolution of the magnetic states is consistent with the theoretical prediction. Meanwhile, the spatial RMCD intensity maps under a low field reveal uniform magnetization without distinct magnetic domains across each area (tens of micrometers), implying that the domain structure is not dominant under our experimental conditions and may not be the cause of vanishing QAHE at a low field [24,29].

\section{Thickness-temperature magnetic phase diagram}

Then, we discuss the thickness-dependent temperaturedriven phase diagram from the AFM phase to the PM phase. The height line profiles [Fig. 3(b) and Fig. S4 in the Supplemental Material, Sec. III [30]] are used to clarify the layer number from 1-SL to 9-SL samples shown in Fig. 3(a). Temperature-dependent RMCD measurements of 2-SL $\mathrm{MnBi}_{2} \mathrm{Te}_{4}$ [Fig. 3(c)] reveal that $\mu_{0} H_{1}$ dwindles as the temperature increases and finally turns into PM at about $20 \mathrm{~K}$. The anomalous magnetic hysteresis loop with a coercive field of about $1.1 \mathrm{~T}$ also shrinks with increasing temperature and disappears at about $20 \mathrm{~K}$. In even- $N$ SL samples, the value of $T_{N}$ is estimated to be the temperature when the spin-flop transition disappears (see the cases for 4-SL and 6-SL samples in Fig. S11 of the Supplemental Material, Sec. VII [30]). As for odd- $N$ SL samples, we examine their magnetism by focusing on the RMCD intensity of the center magnetic hysteresis loop, due to the magnetization of the uncompensated layer. A clear magnetic hysteresis loop appears in 3-SL $\mathrm{MnBi}_{2} \mathrm{Te}_{4}$ at $20 \mathrm{~K}$ [Fig. 3(d)] but vanishes at $22 \mathrm{~K}$, indicating a magnetic phase transition. The value of $T_{N}$ can be extracted from the temperature-dependent remnant RMCD signals at $\mu_{0} H=0 \mathrm{~T}$. The temperature-dependent remnant RMCD signals of 1-SL, 3-SL, 5-SL, and 25-SL flakes can be well fitted using the critical power law $\left(1-T / T_{N}\right)^{\beta}$, where $T<T_{N}, T_{N}$, and $\beta$ are two simultaneous fitting parameters [Fig. 3(e)]. The extracted $T_{N}$ values of 1-SL, 3-SL, 5-SL, and 25 -SL flakes are about $15.2 \mathrm{~K}, 22.1 \mathrm{~K}, 23.4 \mathrm{~K}$, and $24.5 \mathrm{~K}$, respectively (the detailed fitting results are shown in Table S1 in the Supplemental Material, Sec. VII [30]), which increases with the sample thickness. The obtained 
(a)

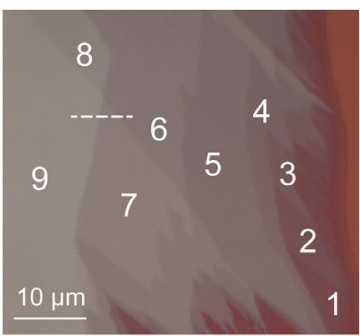

(b)

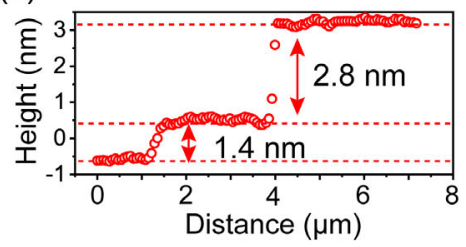

(e)

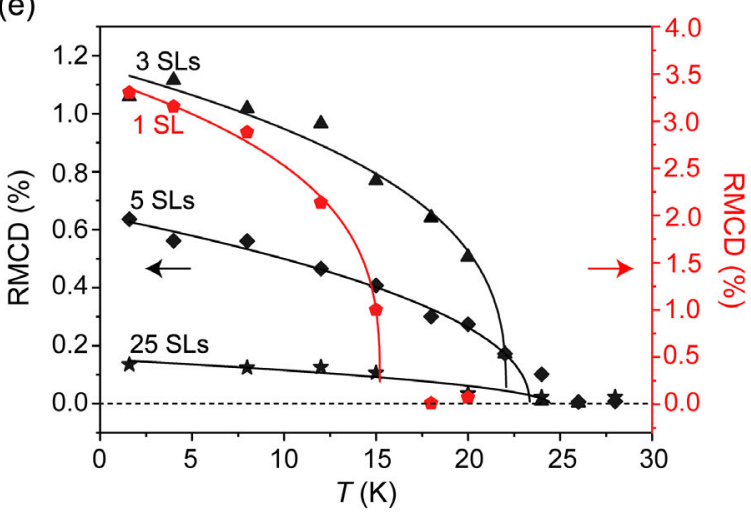

(c) 2 SLs

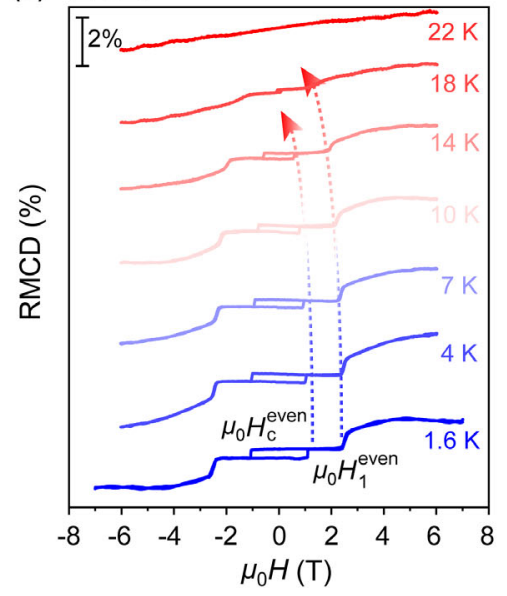

(d) 3 SLs

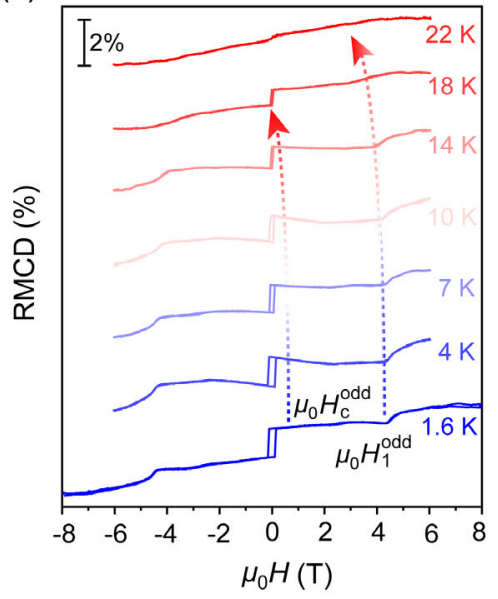

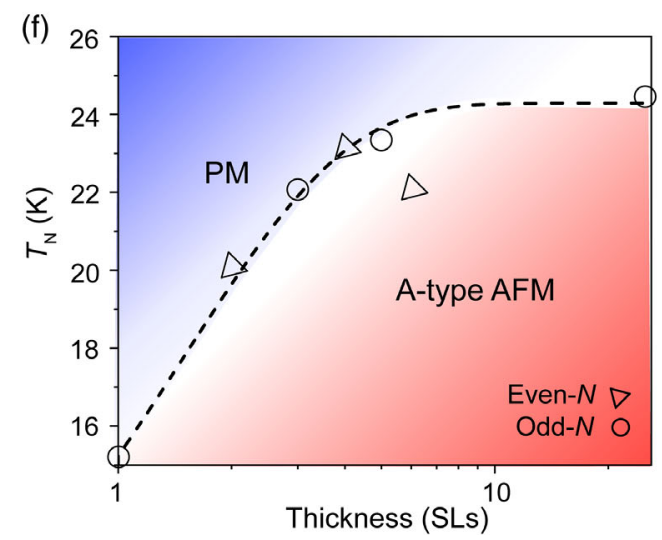

FIG. 3. Temperature-dependent RMCD measurements with different thicknesses and the thickness-temperature phase diagram of $\mathrm{MnBi}_{2} \mathrm{Te}_{4}$. (a) Optical image of the stepped $\mathrm{MnBi}_{2} \mathrm{Te}_{4}$ flakes with $1 \mathrm{SL}$ to $9 \mathrm{SLs}$. (b) Line height profile of the $\mathrm{MnBi}_{2} \mathrm{Te}_{4}$ flakes, along with the white dashed line in panel (a). (c) RMCD sweeps for the 2-SL sample at a temperature range that passes through its $T_{N}$. The spin-flop field $\left(\mu_{0} H_{1}\right)$ decreases as the temperature increases and eventually vanishes at about $20 \mathrm{~K}$. (d) RMCD sweeps for the 3-SL sample at a temperature range that passes through its $T_{N}$. The coercive field and remnant RMCD signal of the magnetic loop decrease as the temperature increases, and both eventually vanish at about $22 \mathrm{~K}$. (e) Remnant RMCD signal as a function of temperature for the selected few- $N$ SL flakes (1 SL, $3 \mathrm{SLs}, 5 \mathrm{SLs}$, and $25 \mathrm{SLs})$. The solid lines are least-squares criticality fits with the form $\left(1-T / T_{N}\right)^{\beta}$, and the black dashed line represents zero RMCD signal. (f) Layer number-temperature phase diagram of the $\mathrm{MnBi}_{2} \mathrm{Te}_{4}$ flakes. PM denotes the region where the flake is paramagnetic; A-type AFM denotes the region where adjacent ferromagnetic SLs couple antiferromagnetically with each other.

$T_{N}$ values (open circles for odd- $N$ SL samples, open triangles for even- $N$ SL samples) are plotted versus SL number [Fig. 3(f)], revealing the boundary between the distinct magnetic phases-PM and A-type AFM. For few$\mathrm{SL} \mathrm{MnBi}_{2} \mathrm{Te}_{4}$, the $T_{N}$ drops from the value of $24.5 \mathrm{~K}$ for a 25-SL sample to $15.2 \mathrm{~K}$ for a $1-\mathrm{SL}$ sample. We ascribe the suppression of $T_{N}$ to the increase in thermal fluctuations as the sample approaches the $2 \mathrm{D}$ limit.

\section{Temperature-field magnetic phase diagrams}

In the above linear chain model, only the ground state is considered, which corresponds to zero temperature. However, the "macrospin" approximation will no longer hold strictly at finite temperatures, so we propose a more precise energy expression of an $N$-layer system,

$$
\begin{aligned}
U_{N}= & \sum_{i=1}^{N-1} \sum_{a, b} J_{a, b} \frac{\vec{M}_{i, a} \cdot \vec{M}_{i+1, b}}{M_{s}^{2}}+\frac{1}{2} \sum_{i=1}^{N} \sum_{a, b} J_{a, b}^{\|} \frac{\vec{M}_{i, a} \cdot \vec{M}_{i, b}}{M_{s}^{2}} \\
& -\frac{K}{2} \sum_{i=1}^{N} \sum_{a}\left(\frac{\vec{M}_{i, a} \cdot \hat{z}}{M_{s}}\right)^{2}-\mu_{0} \vec{H} \cdot \sum_{i=1}^{N} \sum_{a} \vec{M}_{i, a}
\end{aligned}
$$

where $J_{a, b}$ stands for the interlayer interactions (only the interaction between the atoms in the nearest-neighbor layers are counted), $J_{a, b}^{\|}$stands for the intralayer interactions, and $K$ denotes the magnetic anisotropy energy. By utilizing the MF method for intralayer interactions to simplify the model, the spin sites are decoupled, and we can choose one representative spin in each layer to get the $\mathrm{N}$-moment energy, which can be written as 


$$
\begin{aligned}
U_{N}^{\mathrm{MF}, 1}= & \sum_{i=1}^{N-1} J \frac{\left\langle\vec{M}_{i}\right\rangle \cdot \vec{M}_{i+1}+\vec{M}_{i} \cdot\left\langle\vec{M}_{i+1}\right\rangle}{M_{s}^{2}} \\
& +\sum_{i=1}^{N} J \| \frac{\vec{M}_{i} \cdot\left\langle\vec{M}_{i}\right\rangle}{M_{s}^{2}}-\frac{K}{2} \sum_{i=1}^{N}\left(\frac{\vec{M}_{i} \cdot \hat{z}}{M_{s}}\right)^{2} \\
& -\mu_{0} \vec{H} \cdot \sum_{i=1}^{N} \vec{M}_{i}-\sum_{i=1}^{N-1} J \frac{\left\langle\vec{M}_{i}\right\rangle \cdot\left\langle\vec{M}_{i+1}\right\rangle}{M_{s}^{2}} \\
& -\frac{1}{2} \sum_{i=1}^{N} J \frac{\left\langle\vec{M}_{i}\right\rangle \cdot\left\langle\vec{M}_{i}\right\rangle}{M_{s}^{2}},
\end{aligned}
$$

where $J=\sum_{a} J_{a, b}=\sum_{b} J_{a, b}$ represents the effective interlayer interaction, and $J^{\|}=\sum_{a} J_{a, b}^{\|}=\sum_{b} J_{a, b}^{\|}$represents the effective intralayer interaction (see Supplemental Material, Sec. VIII, Part I [30] for mathematical derivations). Previously extracted values of $J$ and $K$ in the linear chain model can be directly used in this model. In order to solve this model, a self-consistent condition that gives the statistical average of the magnetic moment $\left\langle\vec{M}_{i}\right\rangle$ must be applied (see Supplemental Material, Sec. VIII, Part I [30] for the discussion about the self-consistent condition and the technical details about solving this model). Thus, the magnetization under an external magnetic field that varies with temperature can be obtained. It is worth noting that this model is equivalent to the linear chain model at zero temperature (see Supplemental Material, Sec. VIII, Part II [30] for details).

Using this method, we obtain the temperature-field phase diagrams of 2-SL to 6-SL samples (Fig. 4, and Fig. S12 of the Supplemental Material, Sec. VIII, Part III [30]). From temperature-field $\left(T-\mu_{0} H\right)$ phase diagrams, it is clear to see the coincidence of the phase boundaries of A-type AFM/ CAFM/FM between theoretical predictions (white circles and triangles in Fig. 4) and experimental behaviors (grey spheres and triangles with error bars) up to $18 \mathrm{~K}$ for $N=2$ and $22 \mathrm{~K}$ for $N \geq 3$ samples. The spin-flip fields in most measuring results are hard to distinguish experimentally, due to the smooth transition process at $\mu_{0} \mathrm{H}_{2}$ at finite temperatures. As the temperature approaches the Néel temperature, the theoretical prediction gradually deviates from the experimental data because fluctuations of $2 \mathrm{D}$ magnetic systems become more dominant in this region, which is ignored in the MF method. In addition, as the temperature increases, the spin-flop takes place with a smoother transition as we observed experimentally, and the surrounding hysteresis loop narrows (eventually disappears) in the $M-H$ curve at higher temperatures (see Fig. S13 in the Supplemental Material, Sec. VIII, Part III [30] for details). All in all, this model predicts the phase diagram very well below the Néel temperature, showing its capability to trace the properties of $2 \mathrm{D}$ antiferromagnetic materials.

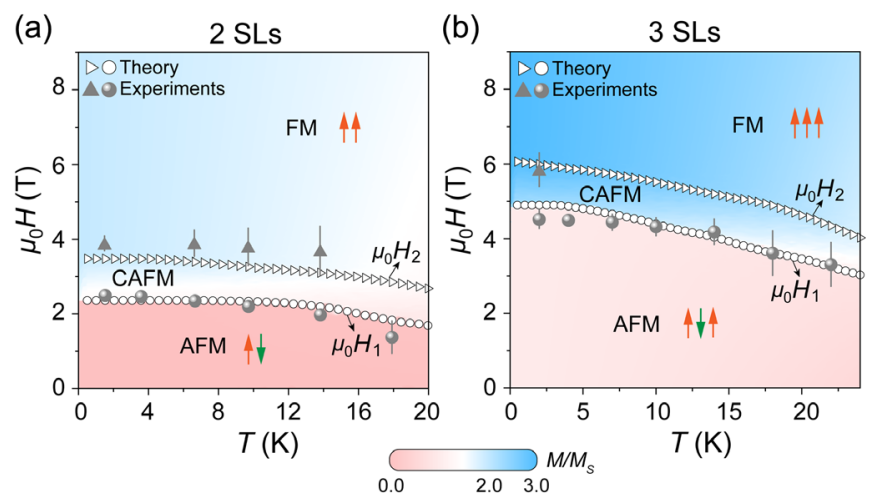

FIG. 4. Temperature-field phase diagrams of 2-SL and 3-SL $\mathrm{MnBi}_{2} \mathrm{Te}_{4}$. (a) Temperature-field phase diagram of 2-SL $\mathrm{MnBi}_{2} \mathrm{Te}_{4}$ determined by MF methods, which is in coincidence with the RMCD measurements. The white circles and triangles represent the calculated spin-flop field $\mu_{0} H_{1}$ and spin-flip field $\mu_{0} H_{2}$, respectively, at various temperatures, showing the boundaries of the A-type AFM/CAFM phase and CAFM/FM phase. The experimental data points are represented using grey spheres and triangles with corresponding error bars. (b) Temperature-field phase diagram of 3-SL $\mathrm{MnBi}_{2} \mathrm{Te}_{4}$, showing the phase boundaries between the uncompensated A-type AFM/CAFM phase and CAFM/FM phase. The scale bar from pink to blue in both graphs shows the magnetization values of $M / M_{s}$ from zero to 3.0. The inset orange $(\uparrow)$ and green $(\downarrow)$ arrows denote the spin orientation of each SL.

\section{CONCLUSIONS}

In summary, we examined layer-dependent magnetism in atomically thin intrinsic magnetic $\mathrm{TI} \mathrm{MnBi}_{2} \mathrm{Te}_{4}$ flakes with varying temperature and applied magnetic field using RMCD spectroscopy. An evident odd-even layer-number effect was observed in thin $\mathrm{MnBi}_{2} \mathrm{Te}_{4}$ flakes, i.e., the oscillations of the coercivity of the hysteresis loop (at $\mu_{0} H_{c}$ ) and the spin-flop transition (at $\mu_{0} H_{1}$ ). The observed anomalous magnetic hysteresis loop in AFM even- $N$ SL samples was probably attributed to surface-induced magnetism; nevertheless, the origin still needs further study, for instance, its relationship with surface topological states and surface structure. The antiferromagnetic linear-chain model provides an excellent quantitative understanding of the experimentally observed odd-even layer-number effect in spin-flop transition field oscillations. It also captures the two-step transitions in even- $N$ SL samples with $N \geq 6$, and the evolution of the magnetic states in $\mathrm{MnBi}_{2} \mathrm{Te}_{4}$ as a function of the magnetic field and number of septuple layers. Based on the MF approach, the temperaturedependent theoretical analyses show the capability to trace the experimentally determined phase diagrams of the few- $N$ SL A-type AFM $\mathrm{MnBi}_{2} \mathrm{Te}_{4}$ in the mid-anisotropy region. The investigation of the magnetic-state evolution with external magnetic field and temperature helps unravel the material's complex magnetic structures and would provide a fundamental understanding for further studying the related quantum states under diverse magnetic phases. 
This work opens more opportunities for further studying the quantum phenomena of magnetic TIs and, plausibly, heterostructures integrating $\mathrm{MnBi}_{2} \mathrm{Te}_{4}$ with other 2D materials that are endowed with more exotic properties in condensed matter physics.

\section{METHODS}

\section{A. Crystal growth and sample preparation}

Single crystals of $\mathrm{MnBi}_{2} \mathrm{Te}_{4}$ were fabricated via a selfflux method. Precisely weighed high-purity manganese powder, bismuth lumps, and tellurium shots were mixed with a molar ratio of $1: 10: 16$, filled into an alumina crucible, and then sealed in a quartz tube under the vacuum at a pressure of less than $5 \mathrm{mTorr}$. The tube was heated to $900{ }^{\circ} \mathrm{C}$ at a rate of $10 \mathrm{~K} / \mathrm{min}$ and kept at this temperature for one day to ensure complete mixing. The tube was then slowly cooled to $600^{\circ} \mathrm{C}$ and quenched with cold water. The shiny, plate-shaped $\mathrm{MnBi}_{2} \mathrm{Te}_{4}$ single crystals with a length of several millimeters were obtained. Thin $\mathrm{MnBi}_{2} \mathrm{Te}_{4}$ flakes with different thicknesses were first mechanically exfoliated on a polydimethylsiloxane (PDMS) substrate and then transferred onto a gold film evaporated on top of a 285- $\mathrm{nm} \mathrm{SiO}_{2} / \mathrm{Si}$ substrate, as reported in previous work in detail [36]. Then, a layer of PMMA was spin coated on the $\mathrm{MnBi}_{2} \mathrm{Te}_{4}$ flakes for protection.

\section{B. RMCD measurements}

The RMCD measurements were performed based on the Attocube closed-cycle cryostat (attoDRY2100) down to $1.6 \mathrm{~K}$ and up to $9 \mathrm{~T}$ in the out-of-plane direction. The sample was moved by an $x y z$ piezo stage (Piezo Positioning Electronic ANC300). A 633-nm HeNe laser with linear polarization was coupled into the system using free-space optics (see Fig. S1 in the Supplemental Material [30] for details). The linearly polarized light was modulated between left and right circular polarization by a photoelastic modulator (PEM) at $50.052 \mathrm{kHz}$ and a chopper at a frequency of $789 \mathrm{~Hz}$. Using a high numerical aperture (0.82) objective, a Gaussian beam with a $2-\mu \mathrm{m}$ diameter spot size was focused onto the sample surface. The reflected light was also collected by the free-space optics and detected by a photomultiplier tube. Because of the polar magneto-optic effect, the magnetization information was detected by the RMCD signal determined by the ratio of the ac component at $50.052 \mathrm{kHz}$ and the ac component at $789 \mathrm{~Hz}$ (both were measured by a two-channel lock-in amplifier Zurich HF2LI).

\section{Raman spectroscopy}

Raman spectra of thick $\mathrm{MnBi}_{2} \mathrm{Te}_{4}$ flakes were obtained using the WITec alpha300 confocal innovation system at room temperature. A 532-nm laser was focused by a $50 \times(0.55$ NA, Zeiss) objective onto the sample, and the resultant Raman signals were detected using a spectrometer with a 1800 groove/mm grating coupled with a charged coupled device (CCD). The temperature-dependent lowfrequency Raman spectra were obtained using free-space optics based on the Attocube closed-cycle cryostat (attoDRY2100). The detailed optical setup is represented in Fig. S2 of the Supplemental Material, Sec. II [30].

\section{ACKNOWLEDGMENTS}

This work was supported by the National Key R\&D Program of China (Grants No. 2018YFA0306900, No. 2017YFA0206301, No. 2019YFA0307800, No. 2019YFA0308000, and No. 2018YFA0704200), the National Natural Science Foundation of China (Grants No. 61521004, No. 11974061, No. U1732162, No. 11974027, and No. 11874405), Beijing Natural Science Foundation (Grant No. Z190011), and the Youth Innovation Promotion Association of CAS (2019007). Y. Y. conceived the project. S. Y. and X.X. performed the measurements. Y.Z. performed the theoretical models and calculations. S. Y., X.X, Y.Z., and Y.Y. analyzed the data and wrote the manuscript. R. N., Y. H., and J. L. prepared the samples. C.X. and X.F.X. grew the $\mathrm{MnBi}_{2} \mathrm{Te}_{4}$ bulk crystals. Y. P., X. C., and X. J. participated in the discussion. All authors discussed the results and contributed to the manuscript.

[1] Y. Tokura, K. Yasuda, and A. Tsukazaki, Magnetic Topological Insulators, Nat. Rev. Phys. 1, 126 (2019).

[2] M.Z. Hasan and C. L. Kane, Colloquium: Topological Insulators, Rev. Mod. Phys. 82, 3045 (2010).

[3] X.-L. Qi and S.-C. Zhang, Topological Insulators and Superconductors, Rev. Mod. Phys. 83, 1057 (2011).

[4] A. M. Shikin, D. A. Estyunin, I. I. Klimovskikh, S. O. Filnov, E. F. Schwier, S. Kumar, K. Myamoto, T. Okuda, A. Kimura, K. Kuroda et al., Nature of the Dirac Gap Modulation and Surface Magnetic Interaction in Axion Antiferromagnetic Topological Insulator $\mathrm{MnBi}_{2} \mathrm{Te}_{4}$, arXiv:2004.04802.

[5] A. Tan, V. Labracherie, N. Kunchur, A. U. B. Wolter, J. Cornejo, J. Dufouleur, B. Büchner, A. Isaeva, and R. Giraud, Metamagnetism of Weakly Coupled Antiferromagnetic Topological Insulators, Phys. Rev. Lett. 124, 197201 (2020).

[6] C.-Z. Chang, J. Zhang, X. Feng, J. Shen, Z. Zhang, M. Guo, K. Li, Y. Ou, P. Wei, L.-L. Wang et al., Experimental Observation of the Quantum Anomalous Hall Effect in a Magnetic Topological Insulator, Science 340, 167 (2013).

[7] R. Yu, W. Zhang, H.-J. Zhang, S.-C. Zhang, X. Dai, and Z. Fang, Quantized Anomalous Hall Effect in Magnetic Topological Insulators, Science 329, 61 (2010).

[8] X.-L. Qi, T. L. Hughes, and S.-C. Zhang, Topological Field Theory of Time-Reversal Invariant Insulators, Phys. Rev. B 78, 195424 (2008).

[9] M. Mogi, M. Kawamura, R. Yoshimi, A. Tsukazaki, Y. Kozuka, N. Shirakawa, K. S. Takahashi, M. Kawasaki, and 
Y. Tokura, A Magnetic Heterostructure of Topological Insulators as a Candidate for an Axion Insulator, Nat. Mater. 16, 516 (2017).

[10] C.-Z. Chang, W. Zhao, D. Y. Kim, H. Zhang, B. A. Assaf, D. Heiman, S.-C. Zhang, C. Liu, M. H. W. Chan, J. S. Moodera, High-Precision Realization of Robust Quantum Anomalous Hall State in a Hard Ferromagnetic Topological Insulator, Nat. Mater. 14, 473 (2015).

[11] I. Vobornik, U. Manju, J. Fujii, F. Borgatti, P. Torelli, D. Krizmancic, Y.S. Hor, R. J. Cava, and G. Panaccione, Magnetic Proximity Effect as a Pathway to Spintronic Applications of Topological Insulators, Nano Lett. 11, 4079 (2011).

[12] D. Xiao, J. Jiang, J.-H. Shin, W. Wang, F. Wang, Y.-F. Zhao, C. Liu, W. Wu, M. H. W. Chan, N. Samarth et al., Realization of the Axion Insulator State in Quantum Anomalous Hall Sandwich Heterostructures, Phys. Rev. Lett. 120, 056801 (2018).

[13] J. Li, Y. Li, S. Du, Z. Wang, B.-L. Gu, S.-C. Zhang, K. He, W. Duan, and Y. Xu, Intrinsic Magnetic Topological Insulators in van der Waals Layered $\mathrm{MnBi}_{2} \mathrm{Te}_{4}$-Family Materials, Sci. Adv. 5, eaaw5685 (2019).

[14] M. M. Otrokov, I. I. Klimovskikh, H. Bentmann, D. Estyunin, A. Zeugner, Z. S. Aliev, S. Gaß, A. U. B. Wolter, A. V. Koroleva, A. M. Shikin et al., Prediction and Observation of an Antiferromagnetic Topological Insulator, Nature (London) 576, 416 (2019).

[15] Y. Gong, J. Guo, J. Li, K. Zhu, M. Liao, X. Liu, Q. Zhang, L. Gu, L. Tang, X. Feng et al., Experimental Realization of an Intrinsic Magnetic Topological Insulator, Chin. Phys. Lett. 36, 076801 (2019).

[16] J. Q. Yan, Q. Zhang, T. Heitmann, Z. Huang, K. Y. Chen, J. G. Cheng, W. Wu, D. Vaknin, B. C. Sales, R. J. McQueeney, Crystal Growth and Magnetic Structure of $\mathrm{MnBi}_{2} \mathrm{Te}_{4}$, Phys. Rev. Mater. 3, 064202 (2019).

[17] H. Li, S. Liu, C. Liu, J. Zhang, Y. Xu, R. Yu, Y. Wu, Y. Zhang, and S. Fan, Antiferromagnetic Topological Insulator $\mathrm{MnBi}_{2} \mathrm{Te}_{4}$ : Synthesis and Magnetic Properties, Phys. Chem. Chem. Phys. 22, 556 (2020).

[18] D. Zhang, M. Shi, T. Zhu, D. Xing, H. Zhang, and J. Wang, Topological Axion States in the Magnetic Insulator $\mathrm{MnBi}_{2} \mathrm{Te}_{4}$ with the Quantized Magnetoelectric Effect, Phys. Rev. Lett. 122, 206401 (2019).

[19] C. Hu, K. N. Gordon, P. Liu, J. Liu, X. Zhou, P. Hao, D. Narayan, E. Emmanouilidou, H. Sun, Y. Liu et al., A van der Waals Antiferromagnetic Topological Insulator with Weak Interlayer Magnetic Coupling, Nat. Commun. 11, 97 (2020).

[20] Z. J. Wu, F. Liu, C. Liu, Y. Wang, C. Li, Y. Lu, S. Matsuishi, and H. Hosono, Toward 2D Magnets in the $\left(\mathrm{MnBi}_{2} \mathrm{Te}_{4}\right)\left(\mathrm{Bi}_{2} \mathrm{Te}_{3}\right)_{n}$ Bulk Crystal, Adv. Mater. 32, 2001815 (2020).

[21] I. I. Klimovskikh, M. M. Otrokov, D. Estyunin, S. V. Eremeev, S. O. Filnov, A. Koroleva, E. Shevchenko, V. Voroshnin, I. P. Rusinov, M. Blanco-Rey et al., Variety of Magnetic Topological Phases in the $\left(\mathrm{MnBi}_{2} \mathrm{Te}_{4}\right)\left(\mathrm{Bi}_{2} \mathrm{Te}_{3}\right)_{m}$ Family, npj Quantum Mater. 5, 54 (2020).

[22] E. D. L. Rienks, S. Wimmer, J. Sánchez-Barriga, O. Caha, P. S. Mandal, and J. Růžička, A. Ney, H. Steiner, V. V. Volobuev, H. Groiss et al., Large Magnetic Gap at the
Dirac Point in $\mathrm{Bi}_{2} \mathrm{Te}_{3} / \mathrm{MnBi}_{2} \mathrm{Te}_{4}$ Heterostructures, Nature (London) 576, 423 (2019).

[23] Y. Deng, Y. Yu, M. Z. Shi, Z. Guo, Z. Xu, J. Wang, X. H. Chen, and Y. Zhang, Quantum Anomalous Hall Effect in Intrinsic Magnetic Topological Insulator $\mathrm{MnBi}_{2} \mathrm{Te}_{4}$, Science 367, 895 (2020).

[24] C. Liu, Y. Wang, H. Li, Y. Wu, Y. Li, J. Li, K. He, Y. Xu, J. Zhang, and Y. Wang, Robust Axion Insulator and Chern Insulator Phases in a Two-Dimensional Antiferromagnetic Topological Insulator, Nat. Mater. 19, 522 (2020).

[25] J. Ge, Y. Liu, J. Li, H. Li, T. Luo, Y. Wu, Y. Xu, and J. Wang, High-Chern-Number and High-Temperature Quantum Hall Effect without Landau Levels, Natl. Sci. Rev. 7, 1280 (2020).

[26] Z. Fei, B. Huang, P. Malinowski, W. Wang, T. Song, J. Sanchez, W. Yao, D. Xiao, X. Zhu, A. F. May et al., TwoDimensional Itinerant Ferromagnetism in Atomically Thin $\mathrm{Fe}_{3} \mathrm{GeTe}_{2}$, Nat. Mater. 17, 778 (2018).

[27] B. Huang, G. Clark, E. Navarro-Moratalla, D. R. Klein, R. Cheng, K. L. Seyler, D. Zhong, E. Schmidgall, M. A. McGuire, D. H. Cobden et al., Layer-Dependent Ferromagnetism in a van der Waals Crystal Down to the Monolayer Limit, Nature (London) 546, 270 (2017).

[28] K. F. Mak, J. Shan, and D. C. Ralph, Probing and Controlling Magnetic States in 2D Layered Magnetic Materials, Nat. Rev. Phys. 1, 646 (2019).

[29] P. M. Sass, W. Ge, J. Yan, D. Obeysekera, J. J. Yang, and W. $\mathrm{Wu}$, Magnetic Imaging of Domain Walls in the Antiferromagnetic Topological Insulator $\mathrm{MnBi}_{2} \mathrm{Te}_{4}$, Nano Lett. 20, 2609 (2020).

[30] See Supplemental Material at http://link.aps.org/ supplemental/10.1103/PhysRevX.11.011003 for RMCD spectroscopy experimental setup; temperature-dependent Raman spectra of thick $\mathrm{MnBi}_{2} \mathrm{Te}_{4}$ and the low-frequency Raman spectroscopy experimental setup; atomic force microscopy measurements of the exfoliated $\mathrm{MnBi}_{2} \mathrm{Te}_{4}$ flakes; additional RMCD data for 1-SL sample under 532-nm and 633-nm excitations; antiferromagnetic linearchain model; RMCD intensity maps including 3 SLs to 5 SLs; temperature-dependent RMCD measurements and fitting results for $T_{N}$ and $\beta$; and magnetic state evolution at finite temperature predicted by a MF method.

[31] D. S. Lee, T.-H. Kim, C.-H. Park, C.-Y. Chung, Y. S. Lim, W.-S. Seo, and H.-H. Park, Crystal Structure, Properties and Nanostructuring of a New Layered Chalcogenide Semiconductor, $\mathrm{Bi}_{2} \mathrm{MnTe}_{4}$, CrystEngComm 15, 5532 (2013).

[32] Z.S. Aliev, I. R. Amiraslanov, D. I. Nasonova, A. V. Shevelkov, N. A. Abdullayev, Z. A. Jahangirli, E. N. Orujlu, M. M. Otrokov, N. T. Mamedov, M. B. Babanly et al., Novel Ternary Layered Manganese Bismuth Tellurides of the $\mathrm{MnTe}-\mathrm{Bi}_{2} \mathrm{Te}_{3}$ System: Synthesis and Crystal Structure, J. Alloys Compd. 789, 443 (2019).

[33] S. H. Lee, Y. Zhu, Y. Wang, L. Miao, T. Pillsbury, H. Yi, S. Kempinger, J. Hu, C. A. Heikes, P. Quarterman et al., Spin Scattering and Noncollinear Spin Structure-Induced Intrinsic Anomalous Hall Effect in Antiferromagnetic Topological Insulator $\mathrm{MnBi}_{2} \mathrm{Te}_{4}$, Phys. Rev. Research 1, 012011 (2019). 
[34] Z. Wang, M. Gibertini, D. Dumcenco, T. Taniguchi, K. Watanabe, E. Giannini, and A. F. Morpurgo, Determining the Phase Diagram of Atomically Thin Layered Antiferromagnet $\mathrm{CrCl}_{3}$, Nat. Nanotechnol. 14, 1116 (2019).

[35] P. M. Sass, J. Kim, D. Vanderbilt, J. Yan, and W. Wu, Robust A-Type Order and Spin-Flop Transition on the Surface of the Antiferromagnetic Topological Insulator $\mathrm{MnBi}_{2} \mathrm{Te}_{4}$, Phys. Rev. Lett. 125, 037201 (2020).

[36] Y. Huang, Y.-H. Pan, R. Yang, L.-H. Bao, L. Meng, H.-L. Luo, Y.-Q. Cai, G.-D. Liu, W.-J. Zhao, Z. Zhou et al., Universal Mechanical Exfoliation of Large-Area 2D Crystals, Nat. Commun. 11, 2453 (2020). 